\section{A case of delayed bleeding 9 days after endoscopic ultrasound-guided pancreatic pseudocyst drainage}

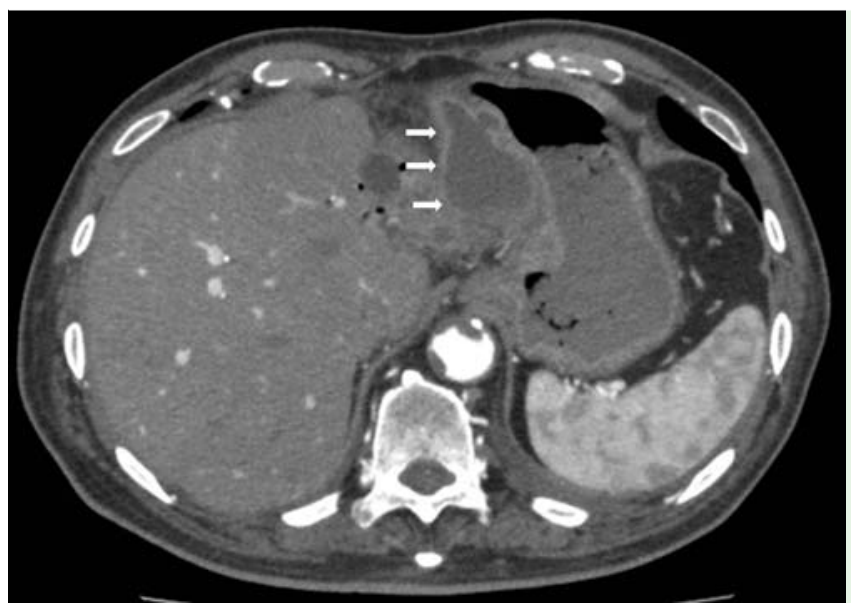

Fig. 1 Computed tomography scan showed a 68-mm multilocular pancreatic pseudocyst (arrow) in contact with the stomach and pancreas.
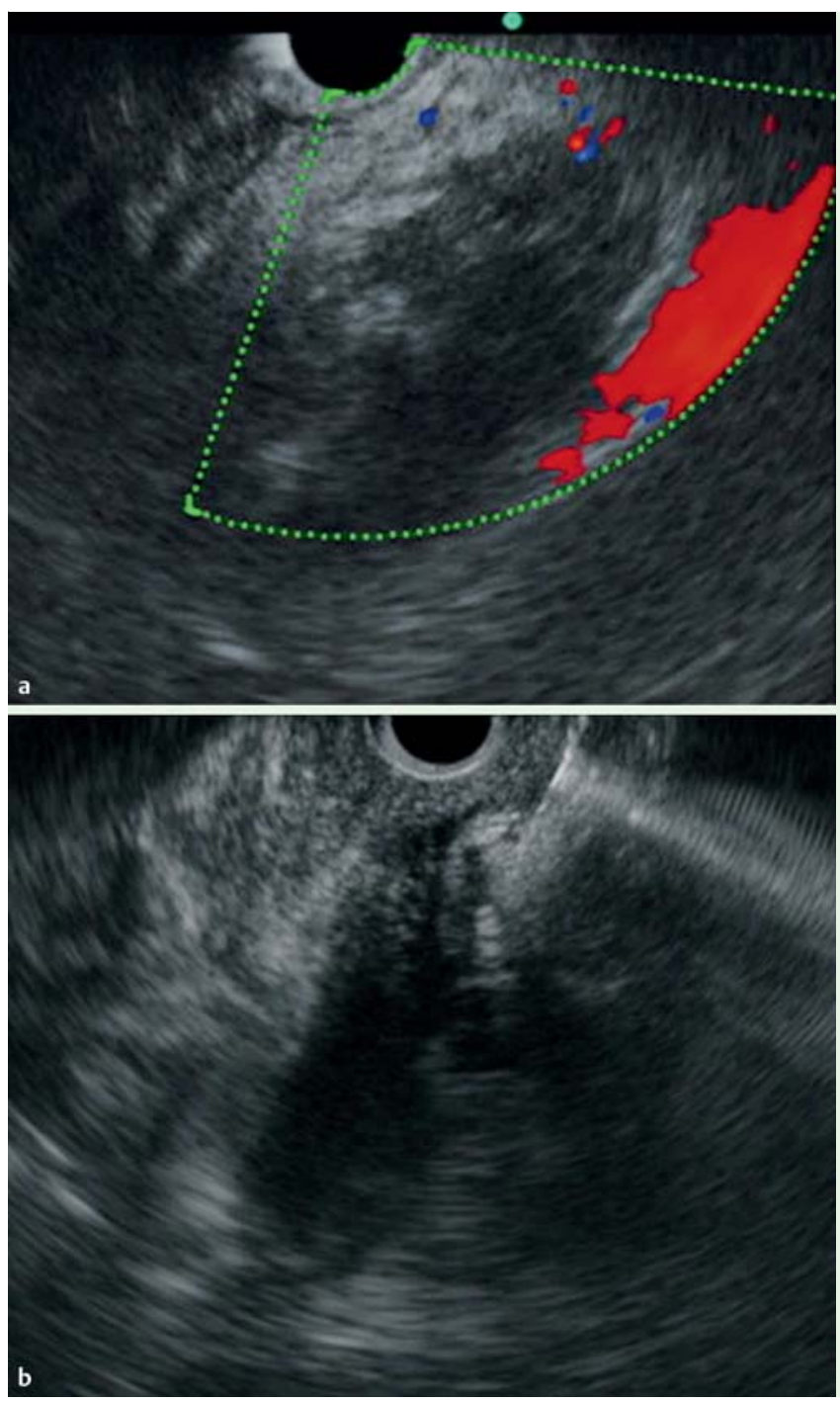

Endoscopic ultrasound-guided pancreatic pseudocyst drainage (EUS-PPD) has become a safe and effective procedure for pancreatic pseudocysts. However, postprocedural complication rates of $8.5 \%-$ $20 \%$ have been reported [1-4]. For delayed complications, only one case of bleeding, which occurred 23 days after EUS-PPD from the transjejunal approach, has been reported [5]. We report a case of bleeding 9 days after EUS-PPD from the transgastric approach.

A 79-year-old man was admitted to our hospital for the treatment of biliary pancreatitis. Heparin was administered because of a history of myocardial infarction. A bile duct stone was successfully removed, but pseudocyst formation occurred ( Fig.1). After 36 days, pseudocyst infection was suspected and EUS-PPD was performed. Heparin was stopped only on the day of the EUS-PPD procedure. Before puncturing the pseudocyst, the absence of intervening blood vessels was confirmed using the color Doppler function ( Fig.2a). The pseudocyst was punctured with a 19-G needle (Sonotip; Medi-Globe GmbH, Rosenheim, Germany) under EUS guidance ( $\bullet$ Fig.2b). Then, an electrical cautery needle (CystGastro set; ENDO-FLEX GmbH, Voerde, Germany) was used to dilate the gastric and cystic wall. Finally, a 5-Fr nasocatheter was inserted into the pseudocyst.

There were no procedure-related adverse events and the symptoms disappeared. Although the serum hemoglobin level did not drop markedly (Day 1: $12.0 \mathrm{mg} / \mathrm{dL}$; Day 8: $11.7 \mathrm{mg} / \mathrm{dL})$, hematemesis was observed 9 days after EUS-PPD and the hemoglobin level dropped to $6.9 \mathrm{mg} / \mathrm{dL}$. Emergency endoscopy showed abundant clots around the pseudocyst fistula ( Fig.3a,b). Angiography showed no aneurysms or contrast medium extravasation ( $\bullet$ Fig. 4$)$.

The late bleeding was possibly caused by the physical stimulus from the inserted stent which caused intracystic mural vessel destruction; the antithrombotic treatment might have been a contributing factor. To our knowledge, this is the first case of delayed bleeding 9 days after EUS-PPD. Sufficient long-term care must be provided after EUS-PPD, particularly in patients receiving antithrombotic treatment.

Endoscopy_UCTN_Code_CPL_1AK_2AG

Competing interests: None 

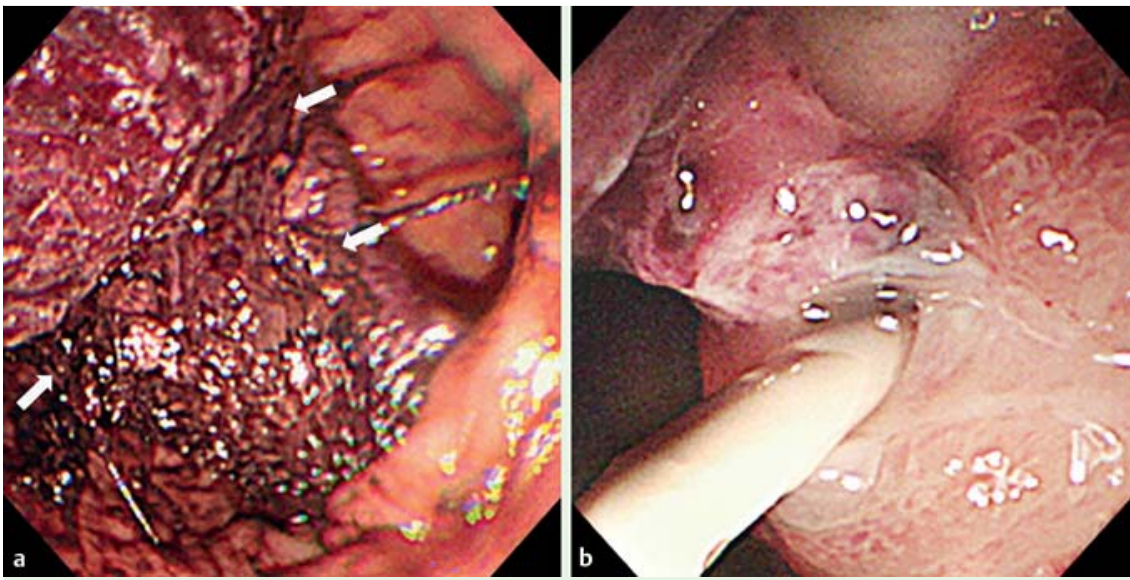

Fig. 3 Emergency endoscopy findings. a, b Abundant clots (arrow) were found around the pseudocyst fistula.

\section{Tomoaki Matsumori, Akio Katanuma, Hiroyuki Maguchi, Kuniyuki Takahashi, Manabu Osanai, Kei Yane, Toshifumi Kin, Ryo Takaki, Kazuyuki Matsumoto, Katsushige Gon, Akiko Tomonari}

Center for Gastroenterology,

Teine-Keijinkai Hospital, Hokkaido, Japan

\section{References}

1 Varadarajulu S, Bang JY, Phadnis MA et al. Endoscopic transmural drainage of peripancreatic fluid collections: outcomes and predictors of treatment in 2011 consecutive patients. J Gastrointest Surg 2011; 15: 2080 2088

2 Park DH, Lee SS, Moon SH et al. Endoscopic ultrasound-guided versus conventional transmural drainage for pancreatic pseudocysts: a prospective randomized trial. Endoscopy 2009; $41: 842-848$

3 Künzli HT, Timmer R, Schwartz MP et al. Endoscopic ultrasonography-guided drainage is an effective and relatively safe treatment for peripancreatic fluid collections in a cohort of 108 symptomatic patients. Eur J Gastroenterol Hepatol 2013; 25: 958-963

4 Puri R, Mishra SR, Thandassery RB et al. Outcome and complications of endoscopic ultrasound guided pancreatic pseudocyst drainage using combined endoprosthesis and naso-cystic drain. J Gastroenterol Hepatol 2012; 27: $722-727$

5 Itoi T, Itokawa $F$, Sofuni A et al. Late bleeding after EUS-guided transjejunal drainage of a pancreatic pseudocyst in a Roux-en-Y patient. Dig Endosc 2011; 23 (Suppl. 01): 51 53

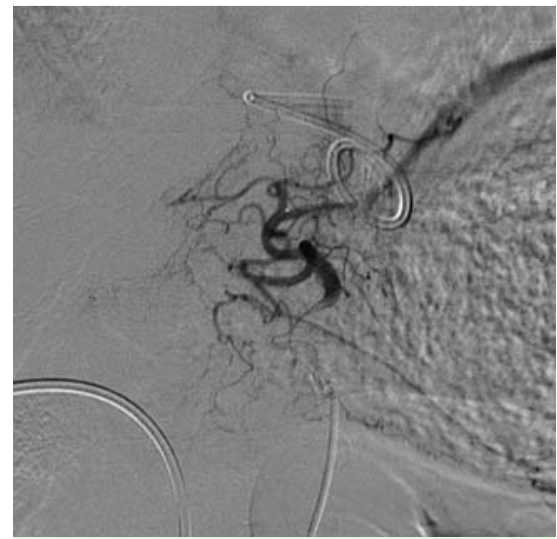

Fig.4 Angiography showed no aneurysms or contrast medium extravasation.

Bibliography

Dol http://dx.doi.org/

10.1055/s-0034-136488

Endoscopy 2014; 46: E245-E246

(c) Georg Thieme Verlag KG

Stuttgart · New York

ISSN 0013-726X

\section{Corresponding author}

\section{Tomoaki Matsumori, MD}

Center for Gastroenterology

Teine-Keijinkai Hospital

1-12-1-40 Maeda

Teine-ku, Sapporo 006-8555

Japan

Fax: +81-11-6852967

tom.matsu1979@gmail.com 